\title{
Efectividad de un programa en la carga de cuidado para cuidadores familiares en tres grupos culturalmente diversos
}

\author{
Effectiveness of a Caregiving Program for Family Caregivers in \\ three Culturally Diverse Groups
}

\section{Eficácia de um programa de cuidados para cuidadores familiares em três grupos culturalmente diversos}

\author{
Yenny Marcela Barreto-Zorza, Enf., MSc * \\ Carolina Enríquez-Guerrero, Enf., MSc ** \\ Vilma Velásquez-Gutiérrez, Enf., MSc ***
}

\begin{abstract}
Resumen
Introducción: El envejecimiento poblacional dado por la transición demográfica y epidemiológica, repercute en la dependencia de las actividades de la vida diaria de los adultos mayores, requiriendo de manera constante los cuidados de personas denominadas "cuidadores", en quienes se genera una carga que afecta su salud física y mental. Objetivo: Evaluar la efectividad de un programa en la carga de cuidado para cuidadores familiares en tres grupos culturalmente diversos. Metodología: Investigación cuantitativa, de tipo analítico, a partir de los resultados de tres estudios cuasiexperimentales de grupos de cuidadores familiares de personas ancianas dependientes funcionalmente. Participaron 88 cuidadores de tres municipios: Bogotá, Cáqueza y Guapi, en un Programa educativo con acciones de cuidado adaptadas culturalmente. La información se recolectó con la Escala de carga de Zarit, antes y después de la implementación del programa, se realizó un análisis con prueba de rango signado de Wilcoxon en cada uno de los tres grupos. Se consideró aspectos éticos. Resultados: El
\end{abstract}

\begin{abstract}
programa educativo para cuidadoras familiares tuvo un efecto estadísticamente significativo en la carga de cuidado de los cuidadores de Cáqueza (valor p 0.014), posiblemente porque el programa responde a una de las necesidades prioritarias de estos cuidadores que es el cuidado de sí mismos, contrario al encontrado en Bogotá y Guapi. Lo que sugiere continuar estudios con programas a cuidadores con perspectiva cultural. Conclusión: El logro de resultados estadísticamente significativos en las cuidadoras de Cáqueza sugiere que ellas cuentan con una mejor red de apoyo familiar y vecinal, por eso asumen la asistencia al programa con mayor dedicación, integralidad y disposición de tiempo. [Barreto-Zorza YM, Enriquez- Guerrero C, Velásquez- Gutiérrez V. Efectividad de un programa en la carga de cuidado para cuidadores familiares en tres grupos culturalmente diversos. MedUNAB 2017; 20(1): 28-38].
\end{abstract}

Palabras clave: Atención de Enfermería; Cultura; Cuidadores; Enfermería en Salud Comunitaria; Educación en Salud; Anciano.

\footnotetext{
* Enfermera, Magister en Salud Pública, Joven Investigadora de Colciencias 2015, profesora de carrera, Escuela de Medicina y Ciencias de la Salud. Universidad del Rosario, Colombia.

** Enfermera, Magister en Enfermería y Epidemiología, Profesora Asistente Universidad Nacional de Colombia

*** Enfermera, Magister en Enfermería y Educación de adultos, Profesora Asociada Universidad Nacional de Colombia.

Correspondencia: Yenny Marcela Barreto-Zorza, dirección de correspondencia: carrera 81b No. 19b-50, Bogotá, Cundinamarca, Colombia. Email: yenny.barreto@urosario.edu.co.
} 


\section{Abstract}

Introduction: The population aging due to the demographic and epidemiological transition implies that elderly people are dependent on activities of daily living, requiring a constant care of people called "caregivers", who generate a burden that affects their physical and mental health. Objective: To evaluate the effectiveness of a caregiving program for family caregivers in three culturally diverse groups. Methodology: This is a quantitative and analytical research based on the results of three quasi-experimental studies of family caregivers groups of functionally dependent elderly people. 88 caregivers from three different municipalities (Bogotá, Cáqueza and Guapi) participated in an educational program with care actions culturally adapted. The data was collected with the Zarit Burden Interview Scale (ZBI); but before and after the implementation of the program, an analysis using the Wilcoxon signed-rank test was performed with each group to compare them. Some ethical aspects were considered as well. Results: The educational program for family caregivers had a statistically significant effect on the burden of care of caregivers from Cáqueza ( $p$ value 0.014 ), possibly because the program responds to one of the priority needs of these caregivers who are taking care of themselves, unlike caregivers from Bogotá and Guapi. The results suggest continuing with more studies and programs to caregivers but with a cultural perspective. Conclusion: Achieving statistically significant results in Cáqueza carers suggests that they have a better family and neighborhood support network, for that reason they assume their attendance to the program with greater dedication, integrality and time availability. [Barreto-Zorza YM, Enriquez- Guerrero C, Velásquez- Gutiérrez V. Effectiveness of a Caregiving Program for Family Caregivers in three Culturally Diverse Groups. MedUNAB 2017; 20(1): 28-38].

Keywords: Nursing Care; Culture; Caregivers; Community Health Nursing; Health Education; Aged.

\section{Introducción}

El aumento de la población adulta mayor refleja una transición demográfica dada por: la caída de la fecundidad y la migración predominante de la población en edad productiva, provocando un déficit en el número de adultos y niños; además, una transición epidemiológica por el control de las enfermedades transmisibles, por mejores condiciones de vida que permiten aumento de la esperanza de vida y envejecimiento poblacional relacionado con la prevalencia de enfermedades crónicas no transmisibles (1). Por tanto, esta población puede ver comprometida su independencia en las actividades de la vida diaria dependiendo de la historia y calidad de vida de cada individuo, esto hace que requieran de manera constante los cuidados de otras personas (2).

La dependencia funcional para las actividades de la vida diaria (bañarse, comer, desplazarse, etc.) requiere cuidados, en la gran mayoría de los casos, por un integrante de la

\section{Resumo}

Introdução: o envelhecimento da população devido à transição demográfica e epidemiológica, afeta a dependência das atividades do cotidiano do idoso, exigindo constantemente o atendimento de pessoas chamadas "cuidadoras", em quem é gerada uma carga que afeta sua saúde física e mental. Objetivo: Avaliar a eficácia de um programa de cuidados para cuidadores familiares em três grupos culturalmente diversos. Metodologia: pesquisa quantitativa, tipo analítico, com base nos resultados de três estudos quase-experimentais de grupos de cuidadores familiares de idosos funcionalmente dependentes. Os participantes foram 88 cuidadores de três municípios: Bogotá, Cáqueza e Guapi, em um programa educacional com ações de cuidados culturalmente adaptadas. A informação foi coletada com o Zarit Load Scale, antes e depois da implementação do programa, foi realizado um teste de classificação Wilcoxon em cada um dos três grupos. Os aspectos éticos foram considerados. Resultados: O programa educacional para cuidadores familiares teve um efeito estatisticamente significativo na carga de cuidados dos cuidadores de Cáqueza (valor p 0.014), possivelmente porque o programa responde a uma das necessidades prioritárias desses cuidadores que cuidam a si mesmos , contrariamente ao encontrado em Bogotá e Guapi. O que sugere continuar estudos com programas para cuidadores com perspectiva cultural. Conclusão: a obtenção de resultados estatisticamente significantes nos cuidadores de Cáqueza sugere que eles tenham uma melhor rede de apoio familiar e de vizinhança, e é por isso que eles cuidam do programa com maior dedicação, completude e disposição do tempo. [Barreto-Zorza YM, Enriquez- Guerrero C, VelásquezGutiérrez V. Eficácia de um programa de cuidados para cuidadores familiares em três grupos culturalmente diversos. MedUNAB 2017; 20 (1): 28-38].

Palavras-chave: Cuidados de Enfermagem; Cultura; Cuidadores; Enfermagem em Saúde Comunitária; Educação em Saúde; Idoso.

familia quien debe asumir estas responsabilidades y es conocido como cuidador familiar, definido por algunos autores como "la persona, habitualmente un familiar directo, que convive y mantiene la responsabilidad de proveer recursos que un paciente incapaz de auto sustentarse necesita"(3).

Zarit identifica la carga generada por la provisión de cuidados como un estado resultante de la acción de cuidar a una persona enferma, dependiente o mayor; un estado que amenaza la salud física y mental del cuidador (4). Es un proceso por el que los cuidadores primarios, evalúan los recursos de que disponen para afrontar las amenazas generadas por los estresores del cuidado (5).

Los cuidadores familiares de adultos mayores asumen la responsabilidad de proporcionar una atención a aquellos que lo necesitan, pero esto puede afectar significativamente su calidad de vida, generando restricciones y/o limitaciones relacionadas con su propia vida. Varios cuidadores perciben 
su salud como regular y esto tiende a empeorar debido al proceso de cuidado de los ancianos, lo que requiere así mismo cuidado de la salud (2), puesto que dejan al margen su autocuidado $(6,7)$ aspectos sociales y económicos de su vida lo que genera un desbalance entre las demandas y las capacidades de los cuidadores, fenómeno conocido como carga del cuidador (8). Es importante tener en cuenta que el cuidador familiar no tiene formación en salud, las mujeres experimentan mayores niveles de carga en comparación con los cuidadores hombres $(7,9)$ y, en su mayoría, son casadas o solteras (10).

Crespo y López (11) refieren que la carga incluye tres factores: el impacto del cuidado, que se relaciona con los efectos que ocasiona la prestación de cuidados al proveedor como son: la falta de tiempo libre, de intimidad, el deterioro de la vida social, entre otras; el factor interpersonal, que está representado por los elementos referidos a la relación del cuidador con la persona cuidada y las expectativas de autoeficacia, que reflejan las creencias del cuidador sobre su capacidad para cuidar a su familiar, el deber de hacerlo, la falta de recursos económicos o la expectativa de no poder continuar cuidándolo por mucho más tiempo. Además, puede repercutir de forma negativa sobre su salud, prevaleciendo problemas de ansiedad y depresión (12).

Durante los últimos 20 años se han creado programas de intervención para ayudar a los cuidadores, sin embargo, estos han sido muy heterogéneos, tanto en los objetivos como en el diseño y en el contenido. En general, estos programas se han desarrollado con el objetivo de obtener un efecto en la sobrecarga o el estrés de los cuidadores y las habilidades de cuidado que tienen cada uno de ellos; han sido desarrollados desde diferentes ámbitos de la salud, lo que revela el interés que genera en los profesionales y la importancia de este problema de salud (13). Con estos programas se ha demostrado que cuando la acción era breve y la muestra de cuidadores heterogénea, la intervención no se mostraba eficaz para disminuir la sintomatología negativa de los cuidadores (14).

Teniendo en cuenta estas dificultades, Zarit (15) analizó la evolución que habían experimentado las intervenciones dirigidas a cuidadores de ancianos dependientes y concluyó que éstas debían planificarse identificando la multidimensionalidad de la sobrecarga y el estrés de los cuidadores, la problemática a la que se dirigía la intervención y utilizando los instrumentos de valoración adecuados; adicionalmente, la literatura refiere que se hace imprescindible seguir explorando esta área de investigación, diseñando intervenciones innovadoras y centradas en las necesidades individuales de los cuidadores de ancianos dependientes que viven en el ámbito domiciliario.

Otros estudios de intervención realizados con cuidadores familiares están dirigidos en su mayoría a aquellos cuidadores de personas con algún tipo de discapacidad cognitiva, las intervenciones en este caso son dirigidas a una terapia cognitivo conductual y un programa de solución de problemas, cuyos resultados destacan que las intervenciones psicoeducativas varían su efecto en los cuidadores en función de los contenidos de dichos programas y se obtienen mejores resultados cuando estos se enfocan en las necesidades o problemas de los cuidadores, reduciendo significativamente sus niveles de ansiedad y mejorando la calidad del sueño con respecto a aquellos que no habían recibido este tratamiento. Cuando los programas se enfocaban en la actividad física mejoraron la adherencia a la práctica de ejercicio físico, así como reducían sus niveles de depresión y carga percibida (16).

Partiendo de esto, y dado que son pocos los estudios de intervención realizados con cuidadores de personas mayores con dependencia funcional por discapacidad física o visual, se decidió practicar un programa educativo a estas personas buscando afectar la carga de cuidado, en cuidadores de los municipios: Bogotá (localidad de Kennedy), Cáqueza y Guapi, que presentan las siguientes características:

En Bogotá, la localidad de Kennedy se encuentra ubicada al suroccidente de la ciudad, población total para el 2015 de 1'030,623 habitantes, con predominio de mujeres (51.3\%). Se evidencia una pirámide regresiva, observando un proceso transicional de envejecimiento. Según la Encuesta Multipropósito del año 2011 efectuada por la Secretaría Distrital de Planeación (SDP) y el DANE el 5.1\%, de los habitantes de la localidad son pobres de acuerdo a las Necesidades Básicas Insatisfechas (NBI). El perfil de morbilidad evidencia el aumento de enfermedades crónicas como hipertensión esencial primaria y enfermedad pulmonar obstructiva crónica.

Kennedy es la segunda localidad receptora (11.5\%) de inmigrantes, que llegan a UPZs que ya tienen problemas de NBI como Patio Bonito y Corabastos, población generalmente compuesta por campesinos o personas que se desplazan por condiciones de pobreza y violencia de las áreas rurales o urbanas de otros municipios del país, comparten algunas problemáticas que afectan la calidad de vida y la salud de las familias, entre ellas: barreras de acceso a la salud, violencia, consumo de sustancias psicoactivas y desempleo(17).

En Cáqueza Cundinamarca, municipio situado a 39 kilómetros de Bogotá, la población total es de 16,467 habitantes, la mayoría habita en la zona rural (60\%) (18), predominan por género los hombres $(51 \%)$, los índices de NBI a nivel municipal, superan los porcentajes departamentales, $46.7 \%$ y $34 \%$ respectivamente. Dentro de las diez causas de morbilidad las enfermedades crónicas y las infecciosas ocupan los primeros lugares, cuya ocurrencia está relacionada, entre otros, con la edad.

Dadas estas características, se puede afirmar que en el municipio predomina la población campesina, término aplicado a las personas que se ocupan en agricultura, 
ganadería, artesanías, dependiendo sobre todo del trabajo familiar(19).

El municipio de Guapi, ubicado en el departamento del Cauca (sur de Colombia), cuenta con una población predominantemente afrocolombiana $(84 \%)$, con un total de 30,424 habitantes, donde predominan las mujeres con $50.2 \%$ (20). Desde el año 2005 el 87.4\% de los habitantes de Guapi presentan NBI, lo que permite inferir que es el segundo municipio más pobre del departamento (21). Las enfermedades que predominan son la hipertensión arterial, enfermedad diarreica aguda (EDA), Infección respiratoria aguda (IRA), malaria, y enfermedades culturales (22). La circunstancia de violencia que atraviesa actualmente esta zona del país ha generado cambios en los roles de la mujer y la participación en el mercado del trabajo, a expensas de una notable precarización de sus condiciones laborales basadas en el rebusque y la informalidad(23).

Por lo anterior, el objetivo de este estudio es evaluar el efecto en la carga de cuidado de un programa educativo para cuidadores familiares de personas ancianas con dependencia funcional en tres grupos culturalmente diversos.

\section{Metodología}

Investigación cuantitativa, de tipo analítico, a partir de los resultados de tres estudios cuasiexperimentales de grupos de cuidadores familiares de personas ancianas dependientes funcionalmente, en los municipios de Bogotá, Cáqueza y Guapi, beneficiarios de programas para personas ancianas.

El proceso para la recolección de la información consideró los siguientes pasos que varían de acuerdo al municipio así:

a) Bogotá, contacto a través del Programa de Discapacidad de la Secretaria de Integración Social de la localidad de Kennedy se tomó en cuenta uno de los grupos; Cáqueza con la Coordinación de Salud Pública de la Alcaldía de Cáqueza, programa adulto mayor; y Guapi a través la Fundación Franciscana Da Amor (FFUNDAMOR) Programa Adulto Mayor; b) invitación a participar a los cuidadores; c) encuentro con los cuidadores y presentación del proyecto; d) elección de participantes y firma del consentimiento informado, y e) aplicación de instrumentos pre y postintervención: Bogotá entre noviembre y diciembre de 2008-2010; Cáqueza entre noviembre y diciembre de 2011 y Guapi entre junio y septiembre de 2010.

Los participantes fueron seleccionados a partir de las listas de beneficiarios de los programas mencionados anteriormente, la población del estudio en los tres grupos era de 180 cuidadores. Teniendo en cuenta los criterios de inclusión, exclusión y participación voluntaria, se tomó una muestra por conveniencia que estuvo conformada por 88 personas distribuidas así: 30 cuidadores de Bogotá, 26 de
Cáqueza y 32 de Guapi, que cumplieron los siguientes criterios de inclusión: ser cuidador familiar de persona anciana con limitación física o visual y que dependa de él para las actividades de la vida diaria; haberlo cuidado por un mes o más, ser mayor de 18 años y ser residente en uno de los tres municipios del estudio. Criterios de exclusión: cuidadores de personas con discapacidad mental.

Todos los cuidadores familiares aceptaron voluntariamente participar en el proyecto y firmaron un consentimiento informado que incluía autorización para tomar fotos, grabar y confidencialidad de sus datos personales.

La intervención efectuada se desarrolló igual en los tres grupos con el programa "Pongámosle color a la vida", se utilizó el mismo constructo para las intervenciones, es decir, en los tres grupos se tuvo el mismo objetivo y las mismas actividades de cada sesión, representadas a través de cinco fases: sensibilización, información culturalmente adaptada, toma de decisiones, compromisos de acción y cierre, únicamente fue necesario adaptar aspectos culturales como música, costumbres y lenguaje.

El programa consta de seis sesiones educativas con un intervalo de tiempo entre cada sesión de un mes en los cuidadores de Bogotá y Cáqueza, y 15 días en los de Guapi. Se trabajaron los temas: redes de apoyo, fortalezas, límites, reconocimiento de la carga de cuidado, identificación de estado de salud física, mental y espiritual.

Es importante aclarar que el desarrollo del programa, al ser diferente en cuanto al intervalo de tiempo entre una sesión y otra, además de efectuarse en distintos años puede constituirse en un sesgo de la información.

\section{Instrumentos}

Fueron aplicados dos instrumentos: la encuesta sociodemográfica a los cuidadores familiares generada por el grupo de investigación y la escala de carga de Zarit, para evaluar la carga de cuidador con una validez de criterio de $r$ $=0.92(\mathrm{p}<0.001$ en el ámbito internacional). En Colombia cuenta con una sensibilidad del $81.58 \%$, una especificidad de $96.35 \%$ con valores predictivos positivos de $75.61 \% \mathrm{y}$ negativos de $97.42 \%$ (24), alfa de Cronbach de 0.881 (25).

Dicho instrumento consta de 22 preguntas tipo Likert de 5 opciones, cuyos resultados se suman en un puntaje total (22110 puntos), este resultado clasifica al cuidador en "ausencia de sobrecarga" ( $\leq 46)$, "sobrecarga ligera" (47$55)$ o "sobrecarga intensa" (56-110).

\section{Análisis de los datos}

El almacenamiento y análisis de la información se efectuó con el software estadístico SPSS versión 17.0. (Licencia 
IBM, asociada a una IP la cual es 168.176.39.1). Se practicó un análisis descriptivo de las variables sociodemográficas y la distribución porcentual de la carga de cuidado obtenidos por la aplicación de la Escala de Zarit a los cuidadores familiares, en los tres grupos culturalmente diversos.

Para la presentación de los resultados se utilizaron varios cálculos como son frecuencias, distribuciones de frecuencias, máximas y mínimas, prueba no paramétrica de Wilcoxon para muestras pareadas, correspondientes a la prueba pre y post en cada uno de los tres grupos.

\section{Consideraciones éticas}

Acorde a lo establecido en la Resolución 8430 de 1993 del Ministerio de Salud de Colombia. Se obtuvo el aval del Comité de Ética de la Facultad de Enfermería de la Universidad Nacional de Colombia en cada uno de los proyectos en los que se trabajaron con los tres grupos culturalmente diversos, se contó con la autorización de la Secretaria de Integración Social de la localidad de Kennedy-Bogotá, Alcaldía de Cáqueza y Fundación Franciscana Da Amor FFUNDAMOR en Guapi y el consentimiento informado de los participantes. Los resultados obtenidos fueron presentados en cada uno de los municipios objeto de análisis de este estudio a las entidades correspondientes.

\section{Resultados}

\section{Características sociodemográficas de los cuidadores}

En los tres grupos culturalmente diversos se observa que más de la tercera parte de las cuidadoras son mujeres, principalmente en el municipio de Guapi (94\%); más de la mitad de cuidadores están entre los 35 y 59 años en los tres grupos (Bogotá 60\%, Cáqueza 54\%, Guapi 59\%), es decir se encuentran en edad económicamente productiva. Se destaca el predominio de cuidadoras casadas en Bogotá (37\%), solteras en Cáqueza (35\%), con una distribución equitativa en Guapi, entre solteras (28\%), casadas (28\%) y en unión libre $(28 \%)$. En cuanto a la escolaridad de las cuidadoras, en Bogo-tá predominan con primaria completa (40\%), más de la mitad en Cáqueza alcanzan solo la primaria incompleta $(54 \%)$ y casi una tercera parte en Guapi son analfabetas $(31 \%)$.

Predomina la afiliación al régimen subsidiado en salud, dados los criterios de inclusión y las condiciones de vulnerabilidad de las tres poblaciones estudiadas (Tabla 1).

Tabla 1. Características sociodemográficas de los cuidadores familiares en tres grupos culturalmente diversos

\begin{tabular}{|c|c|c|c|c|}
\hline \multicolumn{2}{|c|}{ Grupos culturales diversos / Variables } & \multirow{2}{*}{$\begin{array}{c}\text { Bogotá } \\
\text { (Urbanos) } \\
n=30\end{array}$} & \multirow{2}{*}{$\begin{array}{c}\text { Cáqueza } \\
\text { (Campesinos) } \\
n=26\end{array}$} & \multirow{3}{*}{$\begin{array}{c}\text { Guapi } \\
\begin{array}{c}\text { (Afrocolombianos) } \\
\mathrm{n}=32\end{array} \\
\%\end{array}$} \\
\hline & & & & \\
\hline & & $\%$ & $\%$ & \\
\hline \multirow[t]{2}{*}{ Género } & Femenino & 77 & 81 & 94 \\
\hline & Masculino & 23 & 19 & 6 \\
\hline \multirow[t]{3}{*}{ Edad } & 19-34 años & 7 & 8 & 3 \\
\hline & 35-59 años & 60 & 54 & 59 \\
\hline & Mayor a 60 años & 33 & 38 & 38 \\
\hline \multirow[t]{5}{*}{ Estado civil } & Casado & 37 & 27 & 28 \\
\hline & Soltero & 20 & 35 & 28 \\
\hline & Viudo & 3 & 4 & 10 \\
\hline & Divorciado & 10 & 11 & 6 \\
\hline & Unión Libre & 30 & 23 & 28 \\
\hline
\end{tabular}




\begin{tabular}{|c|c|c|c|c|}
\hline \multirow[t]{6}{*}{ Escolaridad } & Sin escolaridad & 7 & 7 & 31 \\
\hline & Primariaincompleta & 27 & 54 & 25 \\
\hline & Primaria completa & 40 & 27 & 9 \\
\hline & Secundaria incompleta & 16 & 12 & 16 \\
\hline & Secundaria Completa & 10 & 0 & 16 \\
\hline & Estudios Superiores & 0 & 0 & 3 \\
\hline \multirow{3}{*}{$\begin{array}{l}\text { Régimen de } \\
\text { afiliación }\end{array}$} & Subsidiado & 70 & 92 & 47 \\
\hline & Contributivo & 23 & 8 & 19 \\
\hline & Ninguno & 7 & 0 & 34 \\
\hline
\end{tabular}

Fuente: Bases de datos Bogotá, Guapi y Cáqueza, resultados del programa de Cuidadores "Pongámosle color a la vida", grupo de Cuidado Cultural de la Salud.

\section{Carga de cuidado}

Al iniciar el programa se encontró que las cuidadoras familiares de los tres grupos culturales diversos presentan una distribución de carga de cuidado de más del $67 \%$, destacándose Cáqueza donde más de la mitad perciben sobrecarga $(58 \%)$.

Al finalizar el programa, se encontró mayor efecto en las cuidadoras de Cáqueza (descendió la sobrecarga de 58\% a 19\%) y en Guapi con disminución de sobrecarga leve (pasó de $38 \%$ a $9 \%$ ), y el aumento de percepción de no sobrecarga (varió de $34 \%$ a $66 \%$ ), a diferencia de las cuidadoras de Bogotá que evidencia aumento de la sobrecarga que pasó de $37 \%$ a $43 \%$. En consecuencia, se evidencian resultados estadísticamente significativos (Tabla 2) solo en las cuidadoras de Cáqueza $(\mathrm{p}=0.014)$.

En la Tabla 3, se describen los resultados pre y pos de los ítems de la escala de Zarit que se afectaron o se mantuvieron después de la intervención (Tabla 3). En el ítem 01 se observa que hay una diferencia en la percepción de los cuidadores frente a la necesidad de ayuda de los adultos mayores que cuidan. Se resalta que en Cáqueza y Guapi antes de la intervención existía esa percepción, a diferencia de Bogotá.

Tabla 2. Resultados del efecto en la carga de cuidador de un programa educativo para cuidadores de personas ancianas con discapacidad y pobreza

\begin{tabular}{|c|c|c|c|c|c|c|}
\hline \multirow[t]{2}{*}{$\begin{array}{l}\text { Grupos culturales/ } \\
\text { nivel de carga }\end{array}$} & \multicolumn{2}{|c|}{$\begin{array}{c}\text { Bogotá } \\
\text { (Urbanos) }\end{array}$} & \multicolumn{2}{|c|}{$\begin{array}{c}\text { Cáqueza } \\
\text { (Campesinos) }\end{array}$} & \multicolumn{2}{|c|}{$\begin{array}{c}\text { Guapi } \\
\text { (Afrocolombianos) }\end{array}$} \\
\hline & $\begin{array}{l}\text { Pre } \\
\%\end{array}$ & $\begin{array}{l}\text { Pos } \\
\%\end{array}$ & $\begin{array}{l}\text { Pre } \\
\%\end{array}$ & $\begin{array}{c}\text { Pos } \\
\%\end{array}$ & $\begin{array}{l}\text { Pre } \\
\%\end{array}$ & $\begin{array}{l}\text { Pos } \\
\%\end{array}$ \\
\hline $\begin{array}{l}\text { No sobrecarga } \\
(<47)\end{array}$ & 33 & 40 & 27 & 42 & 34 & 66 \\
\hline $\begin{array}{l}\text { Sobrecarga leve } \\
(47-55)\end{array}$ & 30 & 17 & 15 & 39 & 38 & 9 \\
\hline $\begin{array}{l}\text { Sobrecarga intensa } \\
(>55)\end{array}$ & 37 & 43 & 58 & 19 & 28 & 25 \\
\hline $\begin{array}{l}\text { Valor P. (prueba de } \\
\text { rango signado de } \\
\text { Wilcoxon) }\end{array}$ & \multicolumn{2}{|c|}{0.361} & \multicolumn{2}{|c|}{0.014} & \multicolumn{2}{|c|}{0.383} \\
\hline
\end{tabular}

Fuente: Bases de datos Bogotá, Guapi y Cáqueza, resultados del programa de Cuidadores "Pongámosle color a la vida". 
Tabla 3. Resultados de la Escala de Zarit por ítems de un programa educativo para cuidadores de personas ancianas con discapacidad y pobreza

\begin{tabular}{|c|c|c|c|c|}
\hline \multirow{2}{*}{$\begin{array}{l}\text { 01. “¿Piensa que su } \\
\text { familiar le pide más } \\
\text { ayuda de la que } \\
\text { realmente necesita?", }\end{array}$} & Pre Test & Nunca $(36.7 \%)$ & Rara vez $(34.6 \%)$ & $\begin{array}{c}\text { Algunas veces } \\
(56.3 \%)\end{array}$ \\
\hline & Pos Test & Nunca $(33.3 \%)$ & $\begin{array}{c}\text { Algunas veces } \\
(34.6 \%)\end{array}$ & $\begin{array}{c}\text { Algunas veces } \\
(46.9 \%)\end{array}$ \\
\hline \multirow{2}{*}{$\begin{array}{l}\text { 02. “¿Piensa que } \\
\text { debido al tiempo que } \\
\text { dedica a su familiar no } \\
\text { tiene suficiente tiempo } \\
\text { para usted?" }\end{array}$} & Pre Test & $\begin{array}{l}\text { Nunca y rara } \\
\text { vez ( } 30 \% \text { en } \\
\text { cada una) }\end{array}$ & $\begin{array}{c}\text { Algunas veces } \\
(26.9 \%)\end{array}$ & $\begin{array}{c}\text { Algunas veces } \\
(43.8 \%)\end{array}$ \\
\hline & Pos Test & $\begin{array}{c}\text { Algunas veces } \\
(43.3 \%)\end{array}$ & $\begin{array}{c}\text { Algunas veces } \\
(42.3 \%)\end{array}$ & $\begin{array}{c}\text { Algunas veces } \\
(31.3 \%)\end{array}$ \\
\hline \multirow[t]{2}{*}{$\begin{array}{l}07 . \text { "¿Tiene miedo por } \\
\text { el futuro de su familiar?" }\end{array}$} & Pre Test & $\begin{array}{l}\text { Nunca } \\
(53.3 \%)\end{array}$ & Siempre $(42.3 \%)$ & Siempre $(43.8 \%)$ \\
\hline & Pos Test & $\begin{array}{c}\text { Algunas veces } \\
(46.7 \%)\end{array}$ & $\begin{array}{l}\text { Nunca } \\
(30.8 \%)\end{array}$ & Siempre $(40.6 \%)$ \\
\hline \multirow{2}{*}{$\begin{array}{l}\text { 08. “¿Piensa que su } \\
\text { familiar depende de } \\
\text { usted?" }\end{array}$} & Pre Test & $\begin{array}{l}\text { Siempre } \\
(63.3 \%)\end{array}$ & Siempre $(53.8 \%)$ & Siempre $(62.5 \%)$ \\
\hline & Pos Test & $\begin{array}{l}\text { Siempre } \\
(46.7 \%)\end{array}$ & Siempre $(42.3 \%)$ & Siempre $(34.4 \%)$ \\
\hline \multirow{2}{*}{$\begin{array}{l}\text { 14. "¿Piensa que su } \\
\text { familiar le considera a } \\
\text { usted la única persona } \\
\text { que le puede cuidar?” }\end{array}$} & Pre Test & $\begin{array}{l}\text { Siempre } \\
(66.7 \%)\end{array}$ & Siempre $(46.2 \%)$ & Siempre $(59.4 \%)$ \\
\hline & Pos Test & $\begin{array}{l}\text { Siempre } \\
(53.3 \%)\end{array}$ & Siempre $(26.9 \%)$ & Siempre $(37.5 \%)$ \\
\hline \multirow{2}{*}{$\begin{array}{l}\text { 18. "¿Desearía poder } \\
\text { dejar el cuidado de su } \\
\text { familiar a otra persona?" }\end{array}$} & Pre Test & $\begin{array}{l}\text { Nunca } \\
(56.7 \%)\end{array}$ & $\begin{array}{l}\text { Nunca } \\
(46.2 \%)\end{array}$ & Nunca $(84.4 \%)$ \\
\hline & Pos Test & $\begin{array}{l}\text { Nunca } \\
(46.7 \%)\end{array}$ & $\begin{array}{l}\text { Nunca } \\
(57.7 \%)\end{array}$ & $\begin{array}{l}\text { Nunca } \\
(75 \%)\end{array}$ \\
\hline \multirow{2}{*}{$\begin{array}{l}\text { 20. "¿Piensa que } \\
\text { debería hacer más por } \\
\text { su familiar?" }\end{array}$} & Pre Test & $\begin{array}{l}\text { Siempre } \\
(36.7 \%)\end{array}$ & Siempre $(38.5 \%)$ & Siempre $(40.6 \%)$ \\
\hline & Pos Test & $\begin{array}{l}\text { Siempre } \\
(46.7 \%)\end{array}$ & Siempre $(34.6 \%)$ & $\begin{array}{c}\text { Algunas veces y } \\
\text { nunca }(28.1 \%)\end{array}$ \\
\hline \multirow{2}{*}{$\begin{array}{l}\text { 21. "¿Piensa que podría } \\
\text { cuidar mejor a su } \\
\text { familiar?" }\end{array}$} & Pre Test & $\begin{array}{c}\text { Algunas veces } \\
(33.3 \%)\end{array}$ & $\begin{array}{c}\text { Bastantes veces } \\
(30.8 \%)\end{array}$ & Siempre (46.9\%) \\
\hline & Pos Test & $\begin{array}{c}\text { Siempre } \\
(40 \%)\end{array}$ & $\begin{array}{c}\text { Algunas veces } \\
(30.8 \%)\end{array}$ & Siempre $(31.3 \%)$ \\
\hline \multirow{2}{*}{$\begin{array}{l}\text { 22. "¿Globalmente, qué } \\
\text { grado de carga } \\
\text { experimenta por el } \\
\text { hecho de cuidar a su } \\
\text { familiar?" }\end{array}$} & Pre Test & $\begin{array}{l}\text { Nunca y rara } \\
\text { vez }(26,7 \%)\end{array}$ & $\begin{array}{l}\text { Algunas veces } \\
(38.5 \%)\end{array}$ & $\begin{array}{l}\text { Nunca } \\
(46.9 \%)\end{array}$ \\
\hline & Pos Test & $\begin{array}{c}\text { Rara vez } \\
(30 \%)\end{array}$ & $\begin{array}{l}\text { Nunca } \\
(38.5 \%)\end{array}$ & Nunca $(40.6 \%)$ \\
\hline
\end{tabular}

Fuente: Bases de datos Bogotá, Guapi y Cáqueza, resultados del programa de Cuidadores "Pongámosle color a la vida". 
Los resultados del ítem 02 pueden deberse a que en las sesiones de intervención se trabaja el tiempo que le dedica el cuidador a sí mismo a través de "límites" y distribución del cuidado con otros miembros de la familia, mediante la reflexión de lo que hacen por sí mismos, es decir, muchos cuidadores podrían haber descubierto que no dedicaban tiempo a sí mismos.

En el ítem 07 se observa que aunque en el ser humano el miedo es un sentimiento normal, y más cuando se piensa en consecuencias fatales de un familiar, este programa contribuye a que los cuidadores reflexionen frente al "apego" que se da ante el adulto mayor que se cuida, y se empiece a pensar un poco más en sí mismo a través del descubrimiento de fortalezas y capacidades.

La dependencia entre el cuidador y el adulto mayor es una característica común en estos grupos poblacionales, generando una respuesta de dependencia mutua que se evidencia en los ítems 08 y 14, sin embargo, los resultados muestran que la intervención efectuada pudo haber contribuido en el hecho de disminuir la percepción de dependencia. Igualmente se ve expresado en el ítem 18 donde a pesar de que la creación de redes es una parte fundamental de la intervención realizada, no fue un punto neurálgico en esta población.

Asimismo, los cuidadores tienen claro que por la falta de recursos económicos, educativos, etc., ellos podrían tener un déficit en las acciones de cuidado que proporcionan, lo que se evidencia en los ítem 20 y 21 , en los cuales es importante resaltar que la intervención aunque no tiene un enfoque para educar al cuidador sobre cómo cuidar mejor a su familiar sí le brindó herramientas para definir hasta qué punto el cuidador puede hacer algo para ayudar a su familiar.

En general, llama la atención que en los tres grupos culturalmente diversos los cuidadores no experimentan como tal una carga de cuidado representado en el ítem 22, esto podría deberse a que estas son acciones de su quehacer diario y parte de su cultura.

\section{Discusión}

De acuerdo con la literatura las mujeres son las que más asumen la responsabilidad del cuidado de un familiar (10, 26-30) tal como se observa en el presente estudio, hallazgo que refuerza el papel social de la mujer históricamente determinado como proveedora de cuidado a los hijos y personas mayores.

En un estudio de revisión de literatura de periodos comprendidos entre 1980 y 2004 se encontró que la edad media de las cuidadoras tiende a situarse entre los 70 y 80 años (31), situación que ha cambiado posiblemente por varios factores como la inclusión laboral, económica, educativa y aspectos culturales que hacen que en municipios como Guapi y Cáqueza no sea una obligación cuidar de su familiar sino una opción de vida. Sin embargo, otros estudios indican que en el mundo las mujeres que desempeñan el rol de cuidado se encuentran en edades entre 23 y 87 años, están en una edad donde comienzan a aparecer síntomas de insatisfacción con respecto a su salud y bienestar y se enfrentan con más frecuencia al abandono temporal o definitivo del trabajo remunerado $(32,33)$, la mayoría de los cuidadores son personas en edad productiva, a quienes posiblemente esta situación les afecta su plan de vida (33).

En general, las cuidadoras poseen un nivel educativo bajo, $(9,25)$ resultado comparable con lo encontrado principalmente en Guapi, y similar al encontrado en un estudio realizado en Brasil con personas que se consideran de raza negra, donde el $67.3 \%$ de las cuidadoras son analfabetas, lo que influye en que estas personas no tengan oportunidades laborales y se dediquen a servicios domésticos y al cuidado del familiar dependiente $(23,32)$.

En cuanto al estado civil otros estudios reportan que la mayoría de mujeres son casadas $(14,33)$ mientras que en este estudio se evidencia que también juegan un rol importante en proporciones similares las solteras y quienes viven en unión libre $(34,35)$, esto puede considerarse dadas las características culturales de los municipios en que se trabajó especialmente Guapi, donde es aceptable que una mujer comparta su pareja con otra mujer y entre ellas mismas se ayuden para el sostenimiento de los hijos, situación concordante con un estudio realizado en el Pacífico Colombiano donde predominan las mujeres solteras y en unión libre (33).

Con respecto a la percepción de la carga del cuidado se evidencia que en la mayoría de estudios transversales las cuidadoras presentan una sobrecarga intensa $(28,36,37)$ resultados concordantes con lo encontrado en la pre prueba de las cuidadoras de Cáqueza y Bogotá en la pos prueba, posiblemente por la percepción que tienen frente al cuidado y el tiempo que llevan cuidando al mismo familiar. $\mathrm{Al}$ comparar los resultados de las cuidadoras de Guapi son proporcionales a los encontrados en Colombia, pero diferentes al estudio realizado en la región Pacífica donde prevalece la sobrecarga intensa (33).

Por lo anterior, es importante considerar que el cuidador es una persona que provee ayuda no remunerada usualmente en el contexto doméstico, invirtiendo grandes cantidades de tiempo y energía durante meses o años, tiene un elevado compromiso por su labor, caracterizada por afecto y atención sin límite de horarios, asiste a la persona dependiente en actividades básicas e instrumentales (38), además muchas personas consideran que el rol de cuidador también les aporta una experiencia positiva, a pesar de las dificultades (16), el cuidado se constituye en una 
experiencia cotidiana del diario vivir y una fuente de cariño y compensación por lo recibido antes por su familiar. La percepción de carga aumenta cuando la decisión de cuidar no es por iniciativa propia del cuidador (39).

Como se indica en la introducción del presente artículo son pocos los estudios de intervención con pre prueba y pos prueba con cuidadores de personas mayores con dependencia funcional (física y visual) como lo afirman López y Crespo en su revisión de literatura "La mayoría de los programas están dirigidos a cuidadores de personas con demencia $(81.5 \%)$. Otros utilizan muestras heterogéneas de cuidadores de mayores con alguna limitación física o enfermedad (e.g., ictus, Parkinson...), entre los que se incluyen en ocasiones también cuidadores de personas con demencia". Sin embargo, al comparar estos resultados con estudios de intervención en cuidadores de personas con otro tipo de dependencia los resultados son concordantes con los encontrados en Bogotá, donde prevalece la sobrecarga intensa $(40,41)$.

Asimismo, estudios de intervención con personas ancianas sobre la variable carga, reportan que desarrollar actividades tanto en el domicilio como en grupos de ayuda y con un seguimiento telefónico durante un amplio periodo de tiempo (hasta 9 años), con énfasis en el ejercicio físico (16), promueven mejoras en la autopercepción del estado de salud, además de facilitar una reducción significativa en el nivel de carga del cuidador y en los síntomas depresivos a largo plazo (42), mostrando una mejoría estadísticamente significativa después de la intervención (valor p: 0.002), en $25 \%$ de los cuidadores existía un riesgo de sobrecarga, mientras que $75 \%$ de los cuidadores presentaban sobrecarga antes de hacer las intervenciones de enfermería (42). Por otro lado, un estudio evidencia que las intervenciones a través de programas educativos efectuadas a los cuidadores con sobrecarga producen también disminución de la depresión y la ansiedad, y a la vez aumentan el bienestar percibido por los cuidadores. (43).

Las acciones de educación lideradas en su mayoría por el área de enfermería (16) disminuyen el nivel de sobrecarga de cuidadores y mayor desarrollo en las habilidades y conocimientos respecto al cuidado de la patología y generalidades de la enfermedad de la persona cuidada. (40). Es importante abordar la educación desde la perspectiva cultural para co-crear diversidad de terapéuticas derivadas del diálogo y la mediación entre los saberes populares y profesionales; sin embargo, a pesar de que esta fue la estrategia implementada en el presente estudio es importante considerar otras metodologías de evaluación de esta variable que pueda estar directamente relacionado con las intervenciones realizadas, de acuerdo con un revisión de literatura "donde no hay contacto con el cuidado, dichas intervenciones no son efectivas" (11).

De igual manera, es importante considerar que una de las preocupaciones de los cuidadores y por las que se genera carga es por el aspecto económico, según Espín (2009) los programas educativos generan cambios importantes en el estado emocional, aunque no son suficientes para producir cambios en dimensiones socioeconómicas del cuidador, por lo que recomienda el uso de otro tipo de intervenciones psicosociales (38). Un estudio mostró que la mayoría de cuidadores no obtienen ingresos, o que lo máximo que recibe es un salario mínimo, que puede justificarse no sólo por el tiempo que necesita para dedicarse a cuidar a su familiar, sino porque además en algunos lugares como por ejemplo Guapi no se tienen muchas oportunidades de trabajo. Es importante considerar que en sondeo realizado por organizaciones comunitarias en la localidad bogotana de Kennedy, las familias provienen principalmente del Chocó, Valle y Nariño, y llegaron a la ciudad por situaciones de desplazamiento, aspecto que contribuye a aumentar la carga de estas poblaciones. Todas las personas que participaron en el estudio padecen pobreza económica, ya sea por el desplazamiento, condiciones de su municipio, etc.

\section{Conclusiones}

La consecución de resultados estadísticamente significativos en las cuidadoras de Cáqueza sugiere que ellas cuentan con una mejor red de apoyo familiar y vecinal, donde asumen la asistencia al programa con mayor dedicación, integralidad y disposición de tiempo, un auténtico respiro, es un día que aprovechan además para cumplir otras actividades de responsabilidad con la familia, desde salud, provisión de alimentos, visitas a familiares, gestiones administrativas con el programa de adulto mayor, que permite el logro de varias metas o responsabilidades como la aceptación de la familia para su participación en el programa.

Es importante considerar que si bien las acciones educativas en los estudios de intervención son las que generan mejores efectos, estas implican en su mayoría que el cuidador se desplace de su casa a un punto de encuentro, lo que produce en él angustia de dejar a su familiar en casa "solo", y en muchas ocasiones el cuidador puede ser interrumpido por las necesidades del enfermo, por lo que las sesiones del programa no suponen un momento de respiro o de desconexión para el cuidador. Se deben adecuar las intervenciones a las características específicas de los cuidadores no necesariamente efectuando el programa de manera permanente en casa porque esto implica que no saldrá de su casa nunca y tampoco se evidenciaría un cambio.

Por otro lado, es importante ejecutar un programa que tenga un enfoque interdisciplinario, ya que el área de enfermería es la que en su mayoría desarrolla este tipo de programas, esto con el fin de conseguir cambios en los cuidadores que realmente generen un impacto positivo a corto, mediano y largo plazo. 


\section{Conflicto de intereses}

Los autores manifiestan no tener conflicto de intereses.

\section{Financiación}

CONVOCATORIA NACIONAL JÓVENES INVESTIGADORES E INNOVADORES 2015 COLCIENCIAS. Código Hermes: 31095

\section{Referencias}

1. Reques- Velasco P. El análisis demográfico del envejecimiento: conceptos, técnicas y métodos. Santander: Servicio de publicaciones de la Universidad de Cantabria; 2006. P. 55-76.

2. Ferraz- dos Anjos K, Narriman R, Boery S, Pereira R. Qualidade de vida de cuidadores familiares de idosos dependentes no domicílio. Texto Contexto Enferm, Florianópolis, 2014; 23(3): 600-8.

3. Martín M, Ballesteros J, Ibarra N, Loizaga C, Serrano B, Larumbe MJ et al. Sobrecarga del cuidador de pacientes con enfermedad de Alzheimer y distrés psíquico. Una asociación relegada en la valoración de las demencias. Actas Esp Psiquiatr 2002; 30: 201-6

4. Flórez GE, Rivas RE, Seguel PF. Nivel de sobrecarga en el desempeño del rol de cuidador familiar de adulto mayor con dependencia severa. Concepción. Cienc. enferm. 2012;(1):29-41, 2012

5. Martín M, Salvadó I, Nadal S, Miji LC, Rico JM, Lanz P, et al. Adaptación para nuestro medio de la Escala de Sobrecarga del Cuidador (Caregiver Burden Interview) de Zarit. Rev Gerontol. 1996; 6:338-46.

6. Baptista BO, Beuter M, Girardon-Perlini NMO, Brondani CM, Budó MLD, Santos NO. A sobrecarga do familiar cuidador no âmbito domiciliar: uma revisão integrativa da literatura. Rev Gaúcha Enferm. 2012; 33(1):147-56.

7. Oliveira DC, D'Elboux MJ. Estudos nacionais sobre cuidadores familiares de idosos: revisão integrativa. Rev Bras Enferm, Brasília. 2012; 65(5):829-38

8. Velásquez V, López L, Barreto Y. Cuidadores familiares campesinos: carga de cuidado, tiempo de cuidado y grado de funcionalidad. Investig Enferm. Imagen Desarr. 2014; 16(2):65-80. doi:10.11144/Javeriana.IE16-2.cfcc

9. Marim CM, Silva V, Taminato M, Barbosa DA. Effectiveness of educational programs on reducing the burden of caregivers of elderly individuals with dementia: a systematic review. Rev Lat Am Enfermagem. 2013; 21 Spec No: 267-75.

10. Montalvo Prieto A, Flórez Torres IE. Características de los cuidadores de personas en situación de cronicidad. Cartagena (Colombia). Un estudio comparativo. Salud Uninorte. 2008; 24 (2):181-190

11. Crespo López M, López Martínez J. El apoyo a los cuidadores de familiares mayores dependientes en el hogar: desarrollo del programa "Cómo mantener su bienestar". Ministerio de Trabajo y Asuntos Sociales. Colección Estudios. EST, Serie Dependencia No. 12006. Madrid: IMSERSO; 2006.

12. Chartrand Vázquez A, Arias Sánchez F. Deterioro psicosomático del cuidador crucial ante la enfermedad discapacitante del adulto mayor. Revista 16 de Abril.
2008. Disponible en: http://www.16deabril.sld.cu/ rev/235/02.pdf

13. Zabalegui Yárnoz A, Navarro Díez M, Cabrera Torres E, Gallart Fernández-Puebla A, Bardallo Porras D, Rodríguez Higueras E, Gual García P et al. Eficacia de las intervenciones dirigidas a cuidadores principales de personas dependientes mayores de 65 años. Una revisión sistemática. Rev Esp Geriatr Gerontol. 2008; 43 (3):157-66.

14. Torres Egea $M^{a}$ Pilar, Ballesteros Pérez Esperanza, Sánchez Castillo Pablo David. Programas e intervenciones de apoyo a los cuidadores informales en España. Gerokomos; 2008 [Consultado 22 Junio 2017]; 19(1): 9-15. Disponible en: http://scielo.isciii.es/ scielo.php?script=sci arttext\&pid=S1134$928 \times 2008000100002 \& \operatorname{lng}=\bar{s}$

15. Zarit SH. Methodological considerations in caregiver intervention and outcome research. En: Light E, Niederehe G, Lebowitz BD, ed. Stress effects on family caregivers of Alzheimer's patients: research and interventions. New York: Springer; 1994. p. 351-69.

16. Madruga Vicente M, Gozalo Delgado M, Gusi Fuertes $\mathrm{N}$, Prieto Prieto J. Efectos de un programa de ejercicio físico a domicilio en cuidadores de pacientes de alzheimer: un estudio piloto. Revista de Psicología del Deporte.2009; 18(2): 255-270.

17. Diagnóstico local con participación social 2012. Localidad de Kennedy territorios saludables localidad de Kennedy Hospital del Sur E.S.E vigencia marzo 2013febrero 2014. [Consultado 2016 agosto 13]. Disponible en: goo.gl/HcPNeQ

18. Alcaldía municipal de Cáqueza. Secretaría de Desarrollo Social y Económico. [Consultado 2016 agosto 13]. Disponible en: http://www.caquezac u n d i n a m a r c a. gov. c o / a p c - a a files/62626464336130623331613939306531/PERFIL EPIDEMIOLOGICO 2008.pdf

19. Naciones Unidas. Assamblea General. Consejo de Derechos Humanos. Declaración sobre los derechos de los campesinos y de otras personas que trabajan en las zonas rurales; 2013 [Consultado 2016 agosto 10]. Disponible en: http://www.ohchr.org/Documents/ HRBodies/HRCouncil/WGPleasants/A-HRC-WG-151-2_sp.pdf

20. DANE, Censo General 2005. Perfil Guapi-Cauca. [Consultado 13/08/2016]. Disponible en: https://www. dane.gov.co/files/censo2005/perfiles/cauca/guapi.pdf

21. Colombia. Ministerio de Trabajo. PNUD. Perfil productivo Municipio Guapi. Insumo para el diseño y alternativas para la generación de empleo a las víctimas de la violencia. Red Ormet 2013. 132 p. [en línea]. [Consultado 2016 julio 15]. Disponible en http://docslide.com.br/documents/perfil-productivo-delmunicipio-guapi.html\#

22. Barreto Zorza YM. Efecto de la propuesta de un programa de atención primaria en salud para familias extensas afrocolombianas, Guapi- Cauca. [Tesis de Maestría]. Universidad Nacional de Colombia. Bogotá: 2014.

23. Programa de las Naciones Unidas para el Desarrollo (PNUD) Colombia. Los afrocolombianos frente a los objetivos de desarrollo del milenio. Resumen ejecutivo. ISBN: 978-958-8447-64-3; [Consultado 2016 agosto 13]. Disponible en: http://www.undp.org/content/dam/ colombia/docs/ODM/undp-co-odmafrocolombianos2012.pdf

24. Vélez- Lopera JM, Berbesí- Fernández D, CardonaArango D, Ordóñez- Molina J. Validación de escalas 
abreviadas de Zarit para la medición de síndrome de cuidador primario del adulto mayor en Medellín. Aten Primaria. 2012; 44(7):411-6.

25. Ruth Santiago, Domínguez Pablo, Peláez- Hernández Benjamín, Viridiana- Rincón Trej, Salazar Santiago, Orea- Tejeda Arturo. Propiedades Psicométricas de la Escala de Carga Zarit para Cuidadores de Pacientes con Insuficiencia Cardíaca. Revista científica y profesiona de la Asociación Latinoamericana para la Formación y la Enseñanza de la Psicología-ALFEPSI. 2016

26. Barrera L, Pinto N, Sánchez B. Habilidad de cuidado de cuidadores familiares de personas con enfermedad crónica: comparación de géneros. Actual. enferm. 2006; 9(2): 9-13

27. Maume DJ. Gender differences in restricting work efforts because of family responsibilities. J Marriage Fam. 2006; 68: 859-869.

28. Gómez- Soto M. Cuidar al cuidador informal. Fundación de la Enfermería de Cantabria. Nuber Científ. 2015; 2(15): 57-62

29. Sánchez B. Habilidad de cuidado de los cuidadores de personas en situación de enfermedad crónica. En: El arte y la ciencia del cuidado. Bogotá: Unibiblos; 2002.p.373-85.

30. López J, Crespo M. Intervenciones con cuidadores de familiares mayores dependientes: una revisión. Psicothema. 2007; 19(1):72-80.

31. Pérez- Jiménez D, Rodríguez- Salvá A, HerreraTravieso DM, García- Roche R, Echemendía- Tocabens $B$, Chang- de la Rosa M. Caracterización de la sobrecarga y de los estilos de afrontamiento en el cuidador informal de pacientes dependientes. Rev Cubana Hig Epidemiol. 2013; 51 (2):174-183

32. Muela- Martínez JA, Torres- Colmenero CJ, PeláezPeláez EM. Comparación entre distintas clasificaciones de las estrategias de afrontamiento en cuidadores de enfermos de Alzheimer. Psicothema. 2002; 14(3):55863

33. Carrillo GM, Chaparro L, Sánchez B. Carga del cuidado en cuidadores familiares de personas con enfermedad crónica en la región Pacífica colombiana. Ciencia y enfermería. 2014; XX (2): 83-91.
34. Félix- Alemán A, Aguilar- Hernández RM, MartínezAguilar ML, Ávila- Alpirez H, Vázquez- Galindo L, Gutiérrez- Sánchez G. Bienestar del cuidador/a familiar adulto mayor con dependencia funcional: una perspectiva de género. Cul Cuid. 2012; 16 (33):81-88

35. Cardona- Arango D, Segura- Cardona AM, BerbesíFernández DY, Ordóñez- Molina J, Agudelo- Martínez A. Características demográficas y sociales del cuidador en adultos mayores. Investig. Andina. 2011; 13 (22):178193.

36. Toro- Moraga YL, Rivas- Riveros E. Cuidadores informales rurales de pacientes dependientes severos. Rev Iberoam Educ Investi Enferm. 2016; 6(1):56-62.

37. Espinoza E, Méndez V, Lara R, Rivera P. Factores asociados al nivel de sobrecarga de los cuidadores informales de adultos mayores dependientes. Theoria. 2009; 18 (1):69-79.

38. Cerquera A, Pabón D. Intervención en cuidadores informales de pacientes con demencia en Colombia: una revisión. Psychol. av. discip. 2014; 8(2):73-81.

39. Rogero- García J. Los tiempos de cuidado: el impacto de la dependencia de los mayores en la vida cotidiana de sus cuidadores. Colección Estudios. EST, Serie Dependencia No. 12012. Madrid: Ministerio de Sanidad y Política Social; 2010. p. 327-50.

40. Leal MI, Sales R, Ibáñez E, Giner J, Leal C. Valoración de la sobrecarga en cuidadores informales de pacientes con esquizofrenia antes y después de un programa psicoeducativo. Actas Esp Psiquiatr 2008; 36(2):63-69.

41. Ostman M. Family burden and participation in care: differences between relatives of patients admitted to psychiatric care for the first time and relatives of readmitted patients. J Psych Mental Health Nurs 2004 11:608-15

42. Sánchez RT, Molina EM, Gómez-Ortega OR Intervenciones de enfermería para disminuir la sobrecarga en cuidadores: un estudio piloto. Rev Cuid 2016; 7(1): 1171-84.

43. Flores G, Arcos M. Afrontamiento y sobrecarga subjetiva de cuidadores de paciente con diagnóstico de demencia. Rev Psicología. 2014; 1(1):41-7. 